\title{
Quantifying the Cosmic Web using the Shapefinder diagonistic
}

\author{
Prakash Sarkar \\ Department of Theoretical Physics, TIFR, Mumbai-400005, India \\ email: prakash@theory.tifr.res.in
}

\begin{abstract}
One of the most successful method in quantifying the structures in the Cosmic Web is the Minkowski Functionals. In 3D, there are four minkowski Functionals: Area, Volume, Integrated Mean Curvature and the Integrated Gaussian Curvature. For defining the Minkowski Functionals one should define a surface. We have developed a method based on Marching cube 33 algorithm to generate a surface from a discrete data sets. Next we calculate the Minkowski Functionals and Shapefinder from the triangulated polyhedral surface. Applying this methodology to different data sets, we obtain interesting results related to geometry, morphology and topology of the large scale structure
\end{abstract}

Keywords. methods: data analysis - galaxies: statistics - large-scale structure of universe

\section{Introduction}

A visual inspection of the galaxy surveys, like SDSS, 2dFGRS etc, reveals that the galaxies are arranged into a magnificent architecture. This architecture, a complex distribution of galaxies in different structural elements (filaments, sheets and clusters), is coined as Cosmic Web. Quantifying the Cosmic Web is a major problem in modern cosmology.

There are many statistical tools to quantify the Cosmic Web. One of the most effective diagnostic to quantify the Cosmic Web is the Minkowski Functionals (henceforth MFs), introduced in Cosmology by Mecke et al.(1994). Sahni et al. (1998) introduced Shapefinders, using the ratio of MFs, to quantify individual structural elements in the Cosmic Web. The 2D version of Shapefinders was introduced by Bharadwaj et al.(2000).

In the present work, we will describe an ansatz to accurately estimate the MFs and hence the shapefinders. This include the construction of smoothed density field from a point distribution and then construct triangulated isodensity surface using Marching cube 33 algorithm (Chernyaev (1995)). We have studied this in details and applied it to SDSS datasets.

\section{Minkowski Functionals and Shapefinders}

In 3D, there are four MFs, namely the Volume $(V)$, Surface Area $(S)$, Integrated mean curvature $(C)$ and $\operatorname{Genus}(G)$. These MFs provide a global characterization of individual structures in the Cosmic Web. Using the above MFs, Sahni et al. (1998) devised shapefinders which include those which have dimension of length as well as those that are dimensionless, to classify the large scale structure. The shapefinder that have dimension of length, $\mathcal{H}_{1}=3 V / S, \mathcal{H}_{2}=S / C, \mathcal{H}_{3}=C / 4 \pi G$, are used to evaluate the size of the structures. Based on $\mathcal{H}_{i}$, the useful dimensionless shapefinders - Planarity $\left(\mathcal{P}=\left(\mathcal{H}_{2}-\mathcal{H}_{1}\right) /\left(\mathcal{H}_{2}+\mathcal{H}_{1}\right)\right)$ and Filamentarity $\left(\mathcal{F}=\left(\mathcal{H}_{3}-\mathcal{H}_{2}\right) /\left(\mathcal{H}_{3}+\mathcal{H}_{2}\right)\right)$ are defined to quantify the shape of $3 \mathrm{D}$ objects. 
It is interesting to note that $\mathcal{P}, \mathcal{F} \leqslant 1$. The dimensionless shapefinders $(\mathcal{P}, \mathcal{F})$ along with the $\operatorname{Genus}(G)$ gives the complete information about the shape and the topology of the structure.

For estimating MFs the first basic step is to construct a isodensity surface. For that, we require to construct smoothed density field defined on a rectangular grid from point distribution. Then given a density threshold $\left(\rho_{T H}\right)$, one can construct a triangulated surface using Marching Cube-33 algorithm. For these triangulated surfaces, the MFs can be evaluated by the following equations (also see Sheth (2006) for details):

(a) The Volume is given by $V=\sum_{i=1}^{N_{T}} V_{i}=\sum_{i=1}^{N_{T}} \frac{1}{3} S_{i}(\hat{n} \cdot \vec{P})_{i}$ where $V_{i}$ is the volume of the individual tetrahedron, $\hat{n}$ is the normal vector of $i^{t h}$ triangle and $\vec{P}$ is the position vector of the centroid of $i^{t h}$ triangle. $N_{T}$ is the total number of triangles and $S_{i}$ is the area of the $i^{\text {th }}$ triangle.

(b) The Surface Area is given by $S=\sum_{i=1}^{N_{T}} S_{i}$.

(c) The Genus is given by $G=1-\frac{\chi}{2}=1-\frac{N_{T}-N_{E}+N_{V}}{2}$, where $\chi$ is the Euler characteristics, $N_{E}$ and $N_{V}$ denotes the total number of triangle-edges and trianglevertices of the triangulated surface respectively.

(d) The Integrated Mean Curvature is estimated by calculating the curvature tensor per triangle based on vertex normals on the triangulated surface as describe in Rusinkiewicz (2004)

\section{Test with idealised structures}

We first check reliability of the above technique with Standard Eikonal Surfaces. The three different structures that we consider correspond to Spherical, Ellipsoidal and Torus. We have generated a spherical surface by assigning density $(\rho)$ on a grid using the following relations,

$$
\rho(i, j, k)= \begin{cases}\rho_{0} / R & \text { if }(i, j, k) \neq(11,11,11) \\ \rho_{0} & \text { if }(i, j, k)=(11,11,11)\end{cases}
$$

where $R$ is the radius of the sphere. The threshold $\rho_{T H}$ associated with sphere of radius $R$ is $\rho / \rho_{T H}$. Figure 1(a) shows the percentage error in the MFs as a function of radius of sphere. We note that the percentage errors for all radius $(R>1)$ are less than 0.5 for all MFs. The genus estimated is 0 for sphere of all radii.

We next study the accuracy for triaxial ellipsoid, which depending on the relative scales of the three axes $(a, b, c)$ can be prolate, oblate or spherical. We first study the Prolate deformation. We start with $a>b=c$, and then increase the $b$ and $c$-axis, until we get $a=b=c$. Figure 1 (b) shows the percentage error of MFs as they evolve with the dimensionless variable $c / a$. The results clearly demonstrate the matching MFs with the exact value to a remarkable degree of accuracy. At the $c / a \geqslant 0.2$, the percentage error of all MFs is less than $0.7 \%$. Next we study the oblate deformation of the Ellipsoid. We start we $a<<b=c$ and increase $a$ until to a value such that $a=b=c$. As $a$ increases, the planarity starts decreasing. Figure 1(c) shows the variation of MFs with dimensionless variable $a / c$. The figure clearly shows that the MFs obtained using triangulation match the exact value with high degree of accuracy. We find that $C$ is estimate to the worst accuracy with maximum percentage error of $1.2 \%$. The genus estimated is 1 for both types of ellipsoid irrespective of axis length.

We now demonstrate the accuracy of ansatz for Torus deformation. The torus can be describe by three parameters $a, b$ and $c$. We have considered the deformations of a two kind of torus - Circular Torus $(a=c<<b)$ having circular cross-sections and elliptical Torus $(a \neq c<<b)$ having elliptical cross-section. It is an important test, since it contains 


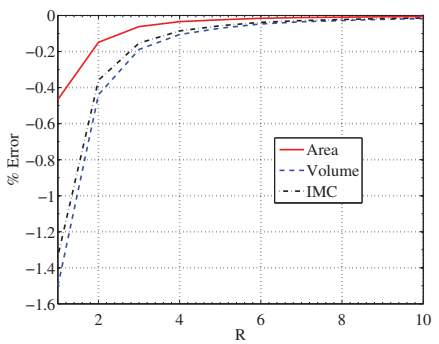

(a) Spherical Surface

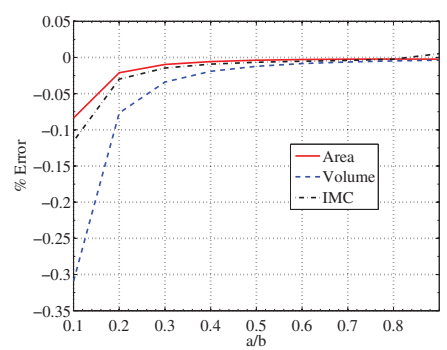

(d) Circular Torus

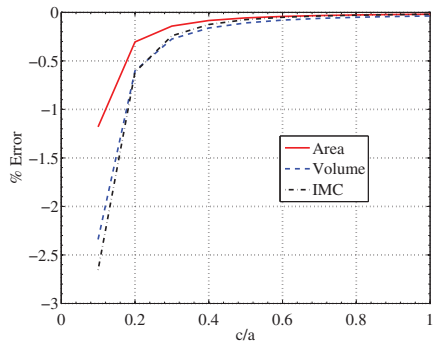

(b) Prolate Ellipsoid

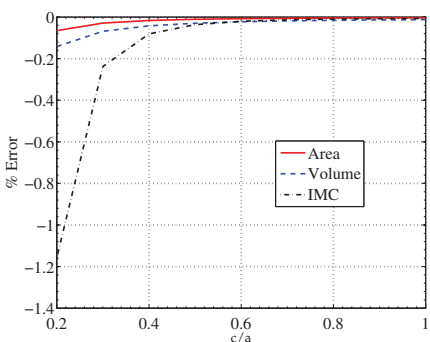

(e) Prolate Torus

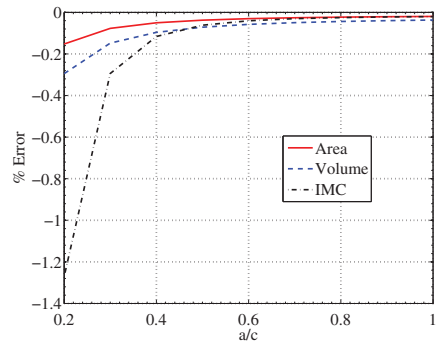

(c) Oblate Ellipsoid

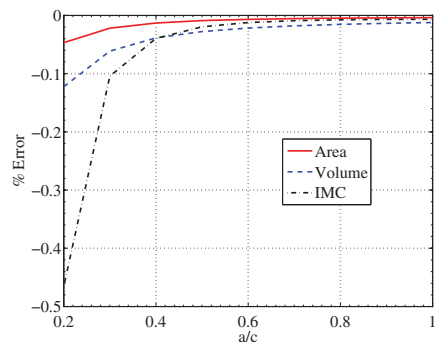

(f) Oblate Torus

Figure 1. The figures shows the variation of percentage error of different MFs with Radius (for sphere) or the ratio of the axis length.

both convex and concave region. For both deformations we have consider $b=20$. Figure $1(\mathrm{~d})$ shows the variation of percentage error for different MFs with $a$. The small error becomes even smaller with increasing $a$. We next study the accuracy for two different types of elliptical Torus -, prolate $(a>c, a=10)$ and oblate $(c>a, c=10)$. Figure $1(\mathrm{e})$ and 1(f) shows the variation of percentage error estimated by ansatz with respect to increasing values $c / a$ and $a / c$ respectively. The results again prove the accuracy of the algorithm. Our results clearly demonstrate the excellent agreement of the calculate value of Minkowski Functionals with the exact analytic formulae.

\section{Results for the SDSS and Conclusion}

We now apply the MFs estimator to quantify the shape of the galaxy distribution in the Sloan Digital Sky Survey Data Release 7. A volume-limited sample of 116, 877 galaxies is constructed by restricting the absolute r-band magnitude brighter than -21.6 . The details of the sample have been discussed in Park et al.(2012). The analysis was carried out by identifying structures using Friend-of-Friend ( $f \circ f$ ) algorithm. The critical linking length is determined by choosing that linking length which results in maximum number of structures. In our case the critical linking length is found out to be $5.6 \mathrm{~h}^{-1} \mathrm{Mpc}$ when the minimum number of member galaxies is set to 20 . There are about 873 structures discovered by fof method. For each structures, we apply a Cloud in Cell (CIC) technique to construct a density field on the grid of size $1 h^{-1}$ Mpc. Next we smooth this density field with a Gaussian kernel of smoothing length $1.9 \mathrm{~h}^{-1} \mathrm{Mpc}$. Now considering a density threshold of 4.15 times the mean density to estimate the Minkowski Functionals and Shapefinders. For comparison we have used 200 mock samples extracted from the Horizon Run 2 (Kim et al.(2011)) simulation. Each Mock samples are analyse in the same way as the SDSS data. This gives the linking lengths of $5.55 \pm 0.13 h^{-1} \mathrm{Mpc}$ from these 200 mock SDSS samples. 


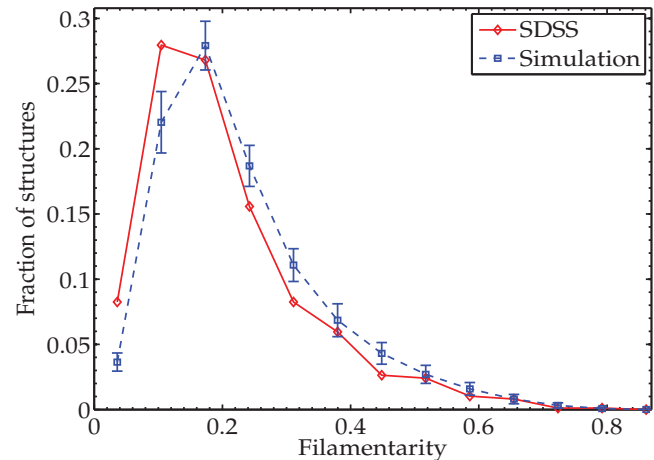

(a) Filamentarity

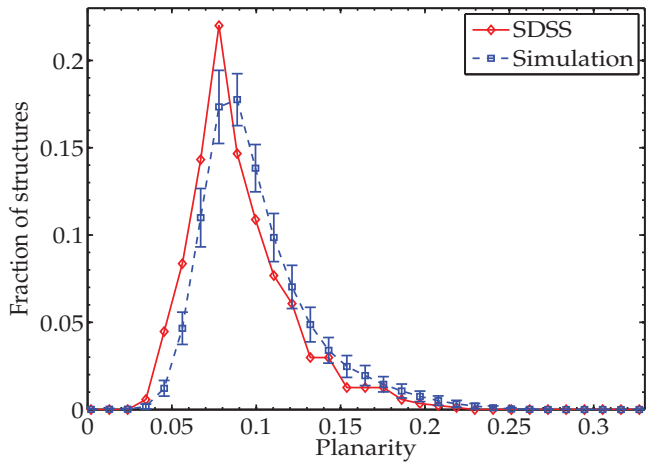

(b) Planarity

Figure 2. This left and right panel show the fraction of structures with Filamentarity and Planarity respectively. The red curve in the figure shows the results of SDSS while the blue dash curve shows the results of Simulations. 200 Mock samples are used to estimate the $1-\sigma$ error-bars.

Figure 2(a) and 2(b) shows the fraction of structures with different Filamentarity and Planarity value respective. The red curve shows that of SDSS and the blue dash curve shows the results for the Mock samples. We find that more than $60 \%$ structures have Filamentarity values in between 0.1 and 0.3 . While more that $80 \%$ of structures are found to have planarity values in the range 0.05 to 0.15 . We find that the results from the Millennium Simulation shifts toward the right to those obtained for the actual SDSS data. This discrepancy is seen for both figures. The origin of this discrepancy is, at the moment, not clear and is an issue we plan to address in future work.

In summary, we can say that the ansatz discussed above gives an accurately estimate the Minkowski Functionals and shapefinders for any arbitrary shape. When apply to structures identified from SDSS galaxies using Friend-of-Friend algorithm, we observe a clear dominance of Filamentarity and Planarity at value 0.2 and 0.1 respectively. We have seen that the Minkowski Functionals and shapefinder estimated using the above method is a robust method to accurately quantify the individual structures in the galaxy distribution.

\section{Acknowledgements}

I am grateful to Prof. Changbom Park for providing the structures identified using Friend-of-Friend algorithm from SDSS and the Simulation data and Prof. Varun Sahni for useful discussions.

\section{References}

Bharadwaj, S., Sahni, V., Sathyaprakash, B. S., Shandarin, S. F., \& Yess, C. 2000, ApJ, 528, 21 Chernyaev, E. V. 1995, Technical report, Marching Cubes 33: Construction of Topologically Correct Isosurfaces

Kim, J., Park, C., Rossi, G., Lee, S. M., Gott, J. R., \& III, 2011, Journal of Korean Astronomical Society, 44, 217

Mecke, K. R., Buchert, T., \& Wagner, H. 1994, A\& A, 288, 697

Park, C., Choi, Y.-Y., Kim, J., et al. 2012, ApJ(letters), 759, L7

Rusinkiewicz, S. 2004, Symposium on 3D Data Processing, Visualization, and Transmission

Sahni, V., Sathyaprakash, B. S., \& Shandarin, S. F. 1998, ApJ(letters), 495, L5

Sheth, J. V., 2006, Ph.D. Thesis 